\title{
Tumor necrosis factor receptor superfamily member 9 is upregulated in the endothelium and tumor cells in melanoma brain metastasis
}

\author{
Patrick N. Harter ${ }^{1,2,3}$, Anna-Eva Blank ${ }^{1,4}$, Benjamin Weide ${ }^{5}$, Rudi Beschorner ${ }^{6}$, Simon Bernatz ${ }^{1}$, Peter Baumgarten ${ }^{1,7}$, \\ Anne K. Braczynski ${ }^{1}$, Elke Hattingen ${ }^{8}$, Michael W. Ronellenfitsch ${ }^{9}$, Herbert Schwarz ${ }^{10}$, Michel Mittelbronn ${ }^{1,2,3}$ \\ ${ }^{1}$ Institute of Neurology (Edinger Institute), Goethe University, 60528 Frankfurt, Germany. \\ ${ }^{2}$ German Cancer Consortium (DKTK), 69120 Heidelberg, Germany. \\ ${ }^{3}$ German Cancer Research Center (DKFZ), 69120 Heidelberg, Germany. \\ ${ }^{4}$ Department of Neuropediatrics, University of Frankfurt, 60590 Frankfurt, Germany. \\ ${ }^{5}$ Department of Dermatology, Eberhardt-Karls University, 72076 Tuebingen, Germany. \\ ${ }^{6}$ Department of Neuropathology, Institute of Pathology and Neuropathology, Eberhardt-Karls University, 72076 Tuebingen, Germany. \\ ${ }^{7}$ Department of Neurosurgery, Goethe University, 60528 Frankfurt, Germany. \\ ${ }^{8}$ Department of Neuroradiology, University of Frankfurt am Main, 60258 Frankfurt, Germany. \\ ${ }^{9} \mathrm{Dr}$. Senckenberg Institute of Neurooncology, University of Frankfurt am Main, 60258 Frankfurt, Germany. \\ ${ }^{10}$ Department of Physiology, Yong Loo Lin School of Medicine, National University of Singapore, Singapore 117456, Singapore.
}

\section{A B S T R A C T}

Aim: The cytokine receptor tumor necrosis factor receptor superfamily member 9 (TNFRSF9) is mainly considered to be a co-stimulatory activation marker in hematopoietic cells. Several preclinical models have shown a dramatic beneficial effect of treatment approaches targeting TNFRSF9 with agonistic antibodies. However, preliminary clinical phase I/II studies were stopped after the occurrence of several severe deleterious side effects. In a previous study, it was demonstrated that TNFRSF9 was strongly expressed by reactive astrocytes in primary central nervous system (CNS) tumors, but was largely absent from tumor or inflammatory cells. The aim of the present study was to address the cellular source of TNFRSF9 expression in the setting of human melanoma brain metastasis, a highly immunogenic tumor with a prominent tropism to the CNS. Methods: Melanoma brain metastasis was analyzed in a cohort of 78 patients by immunohistochemistry for TNFRSF9 and its expression was correlated with clinicopathological parameters including sex, age, survival, tumor size, number of tumor spots, and BRAF V600E expression status. Results: Tumor necrosis factor receptor superfamily member 9 was frequently expressed independently on both melanoma and endothelial cells. In addition, TNFRSF9 was also present on smooth muscle cells of larger vessels and on a subset of lymphomonocytic tumor infiltrates. No association between TNFRSF9 expression and patient survival or other clinicopathological parameters was seen. Of note, several cases showed a gradual increase in TNFRSF9 expression on tumor cells with increasing distance from blood vessels, an observation that might be linked to hypoxia-driven TNFRSF9 expression in tumor cells. Conclusion: The findings indicate that the cellular source of TNFRSF9 in melanoma brain metastasis largely exceeds the lymphomonocytic pool, and therefore further careful (re-) assessment of potential TNFRSF9 functions in cell types other than hematopoietic cells is needed. Furthermore, the hypothesis of hypoxia-driven TNFRSF9 expression in brain metastasis melanoma cells requires further functional testing.

Key words: 4-1BB, brain metastasis, CD137, melanoma, tumor necrosis factor receptor superfamily member 9

\section{INTRODUCTION}

The cytokine receptor tumor necrosis factor receptor

\begin{tabular}{|l|l|}
\hline \multicolumn{2}{|c|}{ Access this article online } \\
\hline Quick Response Code: & \\
\hline & Website: \\
\hline & www.nnjournal.net \\
& DOI: \\
\hline
\end{tabular}

superfamily member 9 (TNFRSF9) (also known as CD137 or 4-1BB) is usually expressed upon cellular activation and acts as a co-stimulatory and the pro-survival molecule in different cellular subsets of the lymphoid and myeloid lineage. ${ }^{[1]}$ Hematopoietic cells which have been activated via TNFRSF9 stimulation have shown an increased antitumor response in various preclinical models. ${ }^{[2,3]}$ This effect was mainly attributed to increased numbers of CD8-positive cytotoxic T-cells as well as antigen-specific memory T-cells.

Corresponding Author: Prof. Michel Mittelbronn, Institute of Neurological (Edinger Institute), Goethe University, Heinrich-Hoffmann-Strasse 7, 60528 Frankfurt (Main), Germany. E-mail: michel.mittelbronn@kgu.de 
Furthermore, tumor-infiltrating lymphocytes (TIL) which have been stimulated with agonistic TNFRSF9 antibodies show a stronger antitumor effect as well as prolonged survival. ${ }^{[4]}$ Therefore, stimulating TNFRSF9 by the use of agonistic antibodies has been proposed as an additional immunotherapeutic approach in cancer treatment-especially for melanoma, which represents one of the most immunogenic tumors-and has already entered clinical trials. ${ }^{[5,6]}$ An alternative approach uses genetically modified human T-cells to express higher TNFRSF9 levels. ${ }^{[7]}$ TNFRSF9 stimulation has been suggested as a treatment for metastatic cancers. ${ }^{[8]}$ However, to date there is only poor data about the distribution of TNFRSF9 in brain metastases, which still constitute one of the most deleterious clinical conditions in tumor patients..$^{[9,10]}$ This data is of importance since preliminary clinical studies targeting TNFRSF9 have already been stopped due to considerable side effects. ${ }^{[11]}$ In a previous study, our group showed that TNFRSF9 was strongly upregulated by reactive astrocytes (so-called gemistocytes) in primary central nervous system (CNS) tumors, whereas both brain tumor cells and TIL were mainly TNFRSF9-negative. ${ }^{[12]}$ Since most studies have only focused on TNFRSF9 expression on hematopoietic cells, there is an urgent need to decipher TNFRSF9 expression on other cell types and different microenvironmental conditions in vivo in more detail. Of note, a recent animal study discovered that TNFRSF9 is also expressed in neural stem cells, in which it induced cell death. ${ }^{[13]}$ The expression of TNFRSF9 on cell types other than hematopoietic cells might be at least partly responsible for side effects in preliminary clinical trials targeting TNFRSF9. Therefore, the aim of our current study was to define the cellular source of TNFRSF9 expression in melanoma brain metastasis, in order to assess the suitability of an anti-TNFRSF9 treatment in this detrimental clinical condition.

\section{METHODS}

\section{Patient data}

The use of human tissue from cases of melanoma brain metastasis and the respective clinical data was approved by the ethical committee of the Eberhard Karls University of Tübingen and Tübingen University Hospital (project no. 408/2013BO2). Our cohort consisted of 78 patients suffering from melanoma brain metastases which underwent neurosurgical resection. A detailed overview of our patient cohort is provided in Table 1. Tissue microarrays were constructed from formalin-fixed and paraffin-embedded tumor samples for immunohistochemical analysis of TNFRSF9 expression. Brain magnetic resonance imaging data was analyzed for metastasis size (diameter) and number. In cases with $>10$ metastases in one patient, the number of metastases was set to 10 for statistical analysis. Patient age at surgery and overall survival after surgery were registered.

\section{Immunohistochemistry}

For immunohistochemistry, a mouse monoclonal antihuman TNFRSF9 antibody (dilution 1:40; clone S16, Novocastra/Leica Microsystems, Germany) was used as previously published. ${ }^{[12]}$ Tissue labeling was performed using the Discovery XT immunohistochemistry system (Ventana Medical Systems, France). A cell conditioning pretreatment was performed for $68 \mathrm{~min}$ followed by a 4 min blocking step with inhibitor D. The primary antibody was applied for $32 \mathrm{~min}$, followed by secondary antibody (Discovery Universal Secondary Antibody) for $32 \mathrm{~min}$. After washing steps, a blocking step with blocker $\mathrm{D}$ for $4 \mathrm{~min}$ and a 16 min incubation with one drop of SA-HRP D were performed. For diaminobenzidine (DAB) visualization, the sections were incubated with one drop of DAB D and one drop of $\mathrm{DAB} \mathrm{H}_{2} \mathrm{O}_{2} \mathrm{D}$ for $8 \mathrm{~min}$, followed by a copper enhancer (Copper D, all Ventana Medical Systems, Tucson, AZ, USA) for 4 min. Specimens were washed, counterstained with hematoxylin and bluing reagent, and mounted. In addition, our cohort was immunohistochemically assessed for BRAF V600E mutations using mouse monoclonal IgG2a antihuman BRAF V600E (dilution 1:100; clone VE1, Spring Bioscience). Images were analyzed and recorded on an Olympus BX-50 microscope (Olympus, Germany).

\section{Scoring}

Tumor necrosis factor receptor superfamily member 9 expression was separately assessed in both tumor and endothelial cells by taking staining intensity and frequency into account, using a previously established protocol. ${ }^{[14,15]}$ The semi-quantitative scores consist of a frequency score ranging from 0 to $4(0=0-1 \%$, $1=2-10 \%, 2=11-25 \%, 3=26-50 \%$, and $4 \geq 50 \%$ of all cells showing positive nuclear staining). Likewise, intensity was recorded in a similar semi-quantitative approach as follows: $0=$ no staining, $1=$ weak staining, $2=$ moderate staining, and $3=$ strong staining. The scores for staining intensity and frequency were multiplied together, so that the final expression cell score reflected both. The evaluation and photographic

\begin{tabular}{lc}
\hline Table I: Patient data & \\
\hline Characteristic & Data \\
\hline Patient age, median (range) & $60(20-83)$ \\
Sex, male/female & $47 / 31$ \\
Number of brain metastases, median (range) & $1(1-10)$ \\
Tumor size in mm, median (range) & $28(5-61)$ \\
BRAF V600E mutation assessed by IHC, yes/no & $38 / 40$ \\
Survival in days, median (range) & $177(17-4166)$ \\
\hline
\end{tabular}

IHC: Immunohistochemistry, BRAF: proto-oncogene B-Raf 
documentation of immunohistochemical staining was performed using an Olympus BX50 light microscope.

\section{Statistical analysis}

The semi-quantitative TNFRSF9 scores were assigned as ordinal scaled response variables and analyzed together with nominal, ordinal, or continuous variables. Nominal and ordinal data was analyzed using a contingency table followed by likelihood ratio and Pearson tests. Survival analyses were performed using Kaplan-Meier and multivariate analyses. In order to compare survival curves, Wilcoxon and log-rank tests were used for censored data. TNFRSF9 expression levels were dichotomized at the median and referred to as low or high. A significance level of alpha $=0.05$ was selected for all tests. Statistical analysis was performed using JMP 11.0.0 software (SAS Institute, Cary, NC, USA).

\section{RESULTS}

Tumor necrosis factor receptor superfamily member 9 is expressed on tumor and endothelial cells in melanoma brain metastasis

Immunohistochemical analyses of our melanoma brain metastasis cohort revealed that reactive astrocytes (gemistocytes) were strongly TNFRSF9-positive, especially at the border between melanoma metastasis and infiltrated CNS tissue, and similarly to our previous findings in a large cohort of primary brain tumors [Figure 1a]. ${ }^{[12]}$ Melanoma cells of brain metastasis showed a very heterogeneous TNFRSF9 staining pattern [Figure 1b]. Frequently, TNFRSF9 expression on melanoma cells became stronger with increasing distance from intra-tumoral blood vessels [Figure 1b], especially in perinecrotic areas. As previously shown, TNFRSF9 was also consistently expressed on smooth muscle cells of larger intra-tumoral blood vessels [Figure 1c]. Of note, TNFRSF9 was also upregulated on endothelial cells of smaller blood vessels within melanoma brain metastasis [Figure 1d]. In addition, a subset of lymphomonocytic infiltrates within melanoma tissue also displayed strong TNFRSF9 expression [Figure 1e]. TNFRSF9 expression on melanoma cells was mainly detected within the cytoplasm [Figure 1f], at the cellular membrane [Figure 1g and h], or both.

Tumor necrosis factor receptor superfamily member 9 expression in melanoma cells does not correlate with expression in endothelial cells within individual melanoma brain metastases

Next, we assessed if TNFRSF9 expression in melanoma brain metastasis was equally upregulated on both tumor and endothelial cells within individual tumors. However, the expression on melanoma cells (median expression score: 4; range: 1-12) was strongly varied in tumors with similar endothelial cell scores (median expression: 3; range: 0-12). No significant correlation between tumor and endothelial cell expression scores was found [Figure 2a]. These findings point to distinct regulatory mechanism of TNFRSF9 in melanoma and endothelial cells. Of note, in cases with an endothelial cell score of $>8$, no melanomas with a tumor cell score $<4$ were found.

Survival of melanoma brain metastasis patients is not associated with tumor necrosis factor receptor superfamily member 9 expression

To address the question of a potential clinicopathological

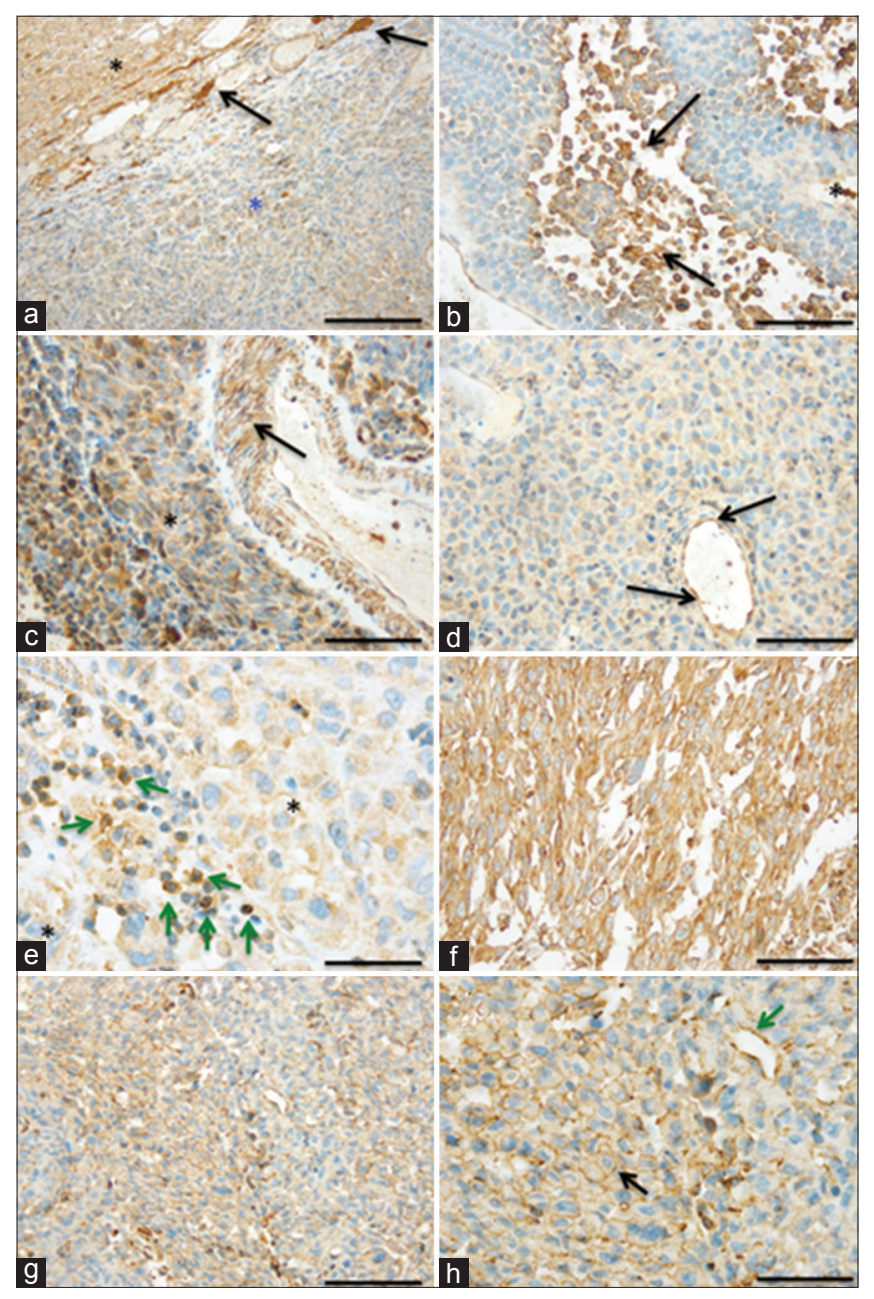

Figure 1: Tumor necrosis factor receptor superfamily member 9 (TNFRSF9) is upregulated on tumor and endothelial cells in melanoma brain metastases. Immunohistochemistry revealing (a) strongly TNFRSF9-positive reactive astrocytes (gemistocytes; arrows) at the border between central nervous system tissue (black asterisk) and melanoma brain metastasis (blue asterisk). (b) Frequently, TNFRSF9 expression on melanoma cells increases (black arrows) with the distance from blood vessels (asterisks). (c) Smooth muscle cells of larger vessels (arrow) within melanoma brain metastasis (asterisk) exhibit strong TNFRSF9-positivity. (d) Apart from melanoma cells, TNFRSF9 is also upregulated on endothelial cells (arrows) of small intra-tumoral blood vessels. (e) Intra-tumoral lymphocytic infiltrates (green arrows) in melanoma brain metastasis (asterisk) also display membranous TNFRSF9-positivity. While some melanoma brain metastases showed strong TNFRSF9 expression (f) both at the cell membrane and within the cytoplasm, $(\mathrm{g})$ others displayed only weak to moderate TNFRSF9 staining at the cell membrane ( $h$ : higher magnification of g. Black arrow: melanoma cells; green arrow: blood vessel). (Scale bars: a: $200 \mu \mathrm{m}$; b, c, d, f, g: $100 \mu \mathrm{m}$; e: $50 \mu \mathrm{m} ; \mathrm{h}: 50 \mu \mathrm{m})$ 
relevance of TNFRSF9 expression in melanoma brain metastasis, we performed Kaplan-Meier survival analysis in our cohort of 78 patients. A survival analysis was performed separately for TNFRSF9 expression on melanoma [Figure 2b] and endothelial [Figure 2c] cells. No significant association of TNFRSF9 expression on melanoma (log-rank test: 0.23; Wilcoxon test: 0.31 ) or endothelial (log-rank test: 0.39; Wilcoxon test: 0.67) cells with patient survival was observed. However, although not showing statistically significant differences, there was a dichotomic tendency for high TNFRSF9 levels and patient survival in melanoma cells as compared to endothelial cells.

Tumor necrosis factor receptor superfamily member 9 levels in melanoma brain metastasis are independent of clinicopathological parameters

The finding of intra-individual differences in TNFRSF9 expression in melanoma brain metastasis [Figure 1b] led to the hypothesis that, in general, tumor size might be associated with increased TNFRSF9 levels due to nutritive changes with increasing tumor volume. However, no significant differences in tumor size were observed with respect to TNFRSF9 scores for melanoma cells [Figure 2d]. In fact, TNFRSF9 scores for melanoma [Figure 2d] and endothelial (data not shown) cells remained quite stable with increasing tumor size. Furthermore, no association of TNFRSF9 expression on melanoma or endothelial cells with patient age, sex, number of brain metastases, or BRAF V600E status was seen (data not shown).

\section{DISCUSSION}

The cellular source of TNFRSF9 expression in melanoma brain metastasis consists of a larger pool than the

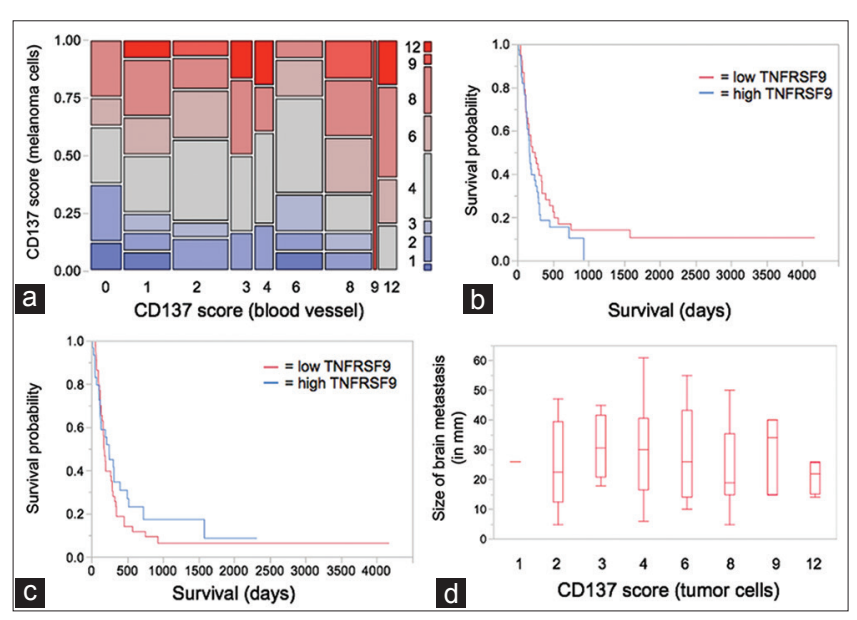

Figure 2: Tumor necrosis factor receptor superfamily member 9 (TNFRSF9) expression in melanoma brain metastasis is independent from clinicopathological data. (a) Contingency analysis of TNFRSF9 expression scores for melanoma and endothelial cells $(n=78)$. (b and $\mathrm{c}$ ) Kaplan-Meier survival curves stratified by median split of TNFRSF9 expression scores for (b) melanoma and (c) endothelial cells. (d) Box-plot diagram of brain metastasis size (in $\mathrm{mm}$ ) versus TNFRSF9 score for melanoma cells typically assessed tumor-infiltrating lymphomonocytic cells. ${ }^{[2,3]}$ In our cohort of 78 melanoma brain metastasis patients, TNFRSF9 expression was frequently detected on both tumor and tumor-associated endothelial cells, but only to a moderate extent on tumor-infiltrating lymphomonocytic cells [Figure 1]. It has been previously shown that TIL that do not express TNFRSF9 display a significantly lower cytolytic antitumor activity. ${ }^{[16]}$ Although melanomas are considered to be highly immunogenic tumors, they possess various strategies to escape from antitumor immune surveillance. ${ }^{[17]}$ However, it is impossible to conclude from our data whether the low TNFRSF9 expression level on tumor-infiltrating immune cells is linked to a primary "underactivation" of the respective cells or an active counter-regulation exerted by melanoma cells. The fact that TNFRSF9 expression on melanoma cells was independent of expression on endothelial cells points to a cell lineage specific upregulation, rather than a general intra-individual regulatory mechanism. Our findings are in line with previous studies that described a selective upregulation of TNFRSF9 on tumor-associated endothelium, whereas endothelial cells from normal control cases remained negative. ${ }^{[18]}$ In addition, TNFRSF9 expression has been discovered on endothelial cells of hypoxic or inflamed blood vessels. ${ }^{[19,20]}$ Our observation that increased TNFRSF9 expression was especially seen in perinecrotic areas-and also with increasing distance from blood vessels-might be related to the fact that TNFRSF9 is also upregulated via hypoxia inducible factor-1 alpha (HIF-1 $\alpha$ ), indicating that hypoxia might also drive its expression on tumor cells. ${ }^{[21]}$ Although it has been demonstrated that HIF1 $\alpha$-related TNFRSF9 upregulation is beneficial for the survival of hematopoietic cells, it can induce cellular apoptosis in other cell types such as liver or tumor cells. ${ }^{[22-24]}$ This might at least partly explain why severe liver toxicity occurred in the first clinical studies targeting TNFRSF9 in humans. ${ }^{[25]}$ Whether enhanced TNFRSF9 expression in perinecrotic areas in melanoma brain metastasis is beneficial or detrimental to the tumor remains an open question. However, a conclusion by analogy can be made, since these areas usually harbor an extremely elevated number of apoptotic cells. Thereby, one can speculate that the upregulation of TNFRSF9 in hypoxic areas is an indication of elevated cell death. Of note, TNFRSF9 expression on either tumor or endothelial cells was not associated with age, sex, patient survival, size or number of brain metastases, or BRAF V600E expression status. Therefore, TNFRSF9 does not serve as a prognostic marker on tumor or endothelial cells per se. Instead, differences in TNFRSF9 expression might reflect inter-individual tumor heterogeneity, including 
alteration of oxygenation or nutrition related to vascularization, rather than a tumor-intrinsic phenomenon. Since we could not define the factors which are responsible for TNFRSF9 upregulation in melanoma brain metastasis, we can only speculate about the underlying reasons. Previous studies have shown that activating protein-1 (AP-1) and NF-kappaB in particular are involved in regulating TNFRSF9. ${ }^{[26]} \mathrm{In}$ contrast to AP-1, NF-kappaB DNA-binding is strongly upregulated in melanomas, indicating that NF-kappaB, but not AP-1, might be one candidate that could drive TNFRSF9 expression in melanomas. ${ }^{[27]}$ However, the relevance of the NF- $\kappa \mathrm{B}$ pathway in stimulating TNFRSF9 expressing in human melanoma brain metastasis definitely needs further investigation.

In summary, our results show that TNFRSF9 is frequently upregulated on both tumor and endothelial cells in melanoma brain metastasis, without being associated with patient survival or any of the clinicopathological parameters assessed in our study. In conclusion, further studies are needed to decipher the exact role of the TNFSF9-TNFRSF9 axis in tumor cells, as well as cells of the tumor micromilieu, in order to understand its link to observed severe side effects in clinical studies targeting TNFRSF9.

\section{REFERENCES}

1. Lee do Y, Choi BK, Lee DG, Kim YH, Kim CH, Lee SJ, Kwon BS. 4-1BB signaling activates the $t$ cell factor 1 effector/B-catenin pathway with delayed kinetics via ERK signaling and delayed PI3K/AKT activation to promote the proliferation of CD8 T Cells. PLoS One 2013;8:e69677.

2. Ju SA, Lee SC, Kwon TH, Heo SK, Park SM, Paek HN, Suh JH, Cho HR, Kwon B, Kwon BS, Kim BS. Immunity to melanoma mediated by $4-1 \mathrm{BB}$ is associated with enhanced activity of tumour-infiltrating lymphocytes. Immunol Cell Biol 2005;83:344-51.

3. Li B, Lin J, Vanroey M, Jure-Kunkel M, Jooss K. Established B16 tumors are rejected following treatment with GM-CSF-secreting tumor cell immunotherapy in combination with anti-4-1BB mAb. Clin Immunol 2007;125:76-87.

4. Chacon JA, Wu RC, Sukhumalchandra P, Molldrem JJ, Sarnaik A, Pilon-Thomas S, Weber J, Hwu P, Radvanyi L. Co-stimulation through 4-1BB/CD137 improves the expansion and function of $\mathrm{CD} 8(+)$ melanoma tumor-infiltrating lymphocytes for adoptive T-cell therapy. PLoS One 2013;8:e60031.

5. Li SY, Liu Y. Immunotherapy of melanoma with the immune costimulatory monoclonal antibodies targeting CD137. Clin Pharmacol 2013;5:47-53.

6. Vinay DS, Kwon BS. Immunotherapy of cancer with 4-1BB. Mol Cancer Ther 2012;11:1062-70.

7. Daniel-Meshulam I, Horovitz-Fried M, Cohen CJ. Enhanced antitumor activity mediated by human 4-1BB-engineered T cells. Int $J$ Cancer 2013;133:2903-13.

8. Schadendorf D, Algarra SM, Bastholt L, Cinat G, Dreno B, Eggermont AM, Espinosa E, Guo J, Hauschild A, Petrella T, Schachter J, Hersey P. Immunotherapy of distant metastatic disease. Ann Oncol 2009;20 Suppl 6:vi41-50.

9. Barnholtz-Sloan JS, Yu C, Sloan AE, Vengoechea J, Wang M, Dignam JJ, Vogelbaum MA, Sperduto PW, Mehta MP, Machtay M,
Kattan MW. A nomogram for individualized estimation of survival among patients with brain metastasis. Neuro Oncol 2012;14: 910-8.

10. Galicich JH, Sundaresan N, Thaler HT. Surgical treatment of single brain metastasis. Evaluation of results by computerized tomography scanning. J Neurosurg 1980;53:63-7.

11. Noji S, Hosoi A, Takeda K, Matsushita H, Morishita Y, Seto Y, Kakimi K. Targeting spatiotemporal expression of CD137 on tumor-infiltrating cytotoxic $\mathrm{T}$ lymphocytes as a novel strategy for agonistic antibody therapy. J Immunother 2012;35:460-72.

12. Blank AE, Baumgarten P, Zeiner P, Zachskorn C, Löffler C, Schittenhelm J, Czupalla CJ, Capper D, Plate KH, Harter PN, Mittelbronn M. Tumor necrosis factor receptor superfamily member 9 (TNFRSF9) is up-regulated in reactive astrocytes in human gliomas. Neuropathol Appl Neurobiol 2014; Epub ahead of print.

13. Yun $\mathrm{CH}$, Lee HM, Lee SC, Kim BS, Park JW, Lee BJ. Involvement of CD137 ligand signaling in neural stem cell death. Mol Cells 2013;36:245-51.

14. Harter PN, Bunz B, Dietz K, Hoffmann K, Meyermann R, Mittelbronn M. Spatio-temporal deleted in colorectal cancer (DCC) and netrin-1 expression in human foetal brain development. Neuropathol Appl Neurobiol 2010;36:623-35.

15. Harter PN, Zinke J, Scholz A, Tichy J, Zachskorn C, Kvasnicka HM, Goeppert B, Delloye-Bourgeois C, Hattingen E, Senft C, Steinbach JP, Plate KH, Mehlen P, Schulte D, Mittelbronn M. Netrin-1 expression is an independent prognostic factor for poor patient survival in brain metastases. PLoS One 2014;9:e92311.

16. Hernandez-Chacon JA, Li Y, Wu RC, Bernatchez C, Wang Y, Weber JS, Hwu P, Radvanyi LG. Costimulation through the CD137/4-1BB pathway protects human melanoma tumor-infiltrating lymphocytes from activation-induced cell death and enhances antitumor effector function. $J$ Immunother 2011;34:236-50.

17. Fourcade J, Zarour HM. Strategies to reverse melanoma-induced T-cell dysfunction. Clin Dermatol 2013;31:251-6.

18. Broll K, Richter G, Pauly S, Hofstaedter F, Schwarz H. CD137 expression in tumor vessel walls. High correlation with malignant tumors. Am J Clin Pathol 2001;115:543-9.

19. Olofsson PS, Söderström LA, Wågsäter D, Sheikine Y, Ocaya P, Lang F, Lang F, Rabu C, Chen L, Rudling M, Aukrust P, Hedin U, Paulsson-Berne G, Sirsjo A, Hansson GK. CD137 is expressed in human atherosclerosis and promotes development of plaque inflammation in hypercholesterolemic mice. Circulation 2008;117:1292-301.

20. Teijeira Á, Palazón A, Garasa S, Marré D, Aubá C, Rogel A, Murillo O, Martinez-Forero I, Lang F, Melero I, Rouzaut A. CD137 on inflamed lymphatic endothelial cells enhances CCL21-guided migration of dendritic cells. FASEB J 2012;26:3380-92.

21. Palazón A, Martínez-Forero I, Teijeira A, Morales-Kastresana A, Alfaro C, Sanmamed MF, Perez-Gracia JL, Penuelas I, Hervas-Stubbs S, Rouzaut A, de Landazuri MO, Jure-Kunkel M, Aragones J, Melero I. The HIF-1a hypoxia response in tumor-infiltrating T lymphocytes induces functional CD137 (4-1BB) for immunotherapy. Cancer Discov 2012;2:608-23.

22. Bellarosa D, Bressan A, Bigioni M, Parlani M, Maggi CA, Binaschi M. SAHA/Vorinostat induces the expression of the CD137 receptor/ligand system and enhances apoptosis mediated by soluble CD137 receptor in a human breast cancer cell line. Int $J$ Oncol 2012;41:1486-94.

23. Gullo C, Koh LK, Pang WL, Ho KT, Tan SH, Schwarz H. Inhibition of proliferation and induction of apoptosis in multiple myeloma cell lines by CD137 ligand signaling. PLoS One 2010;5:e10845.

24. Nagila A, Netsawang J, Suttitheptumrong A, Morchang A, Khunchai S, Srisawat C, Puttikhunt C, Noisakran S, Yenchitsomanus PT, Limjindaporn T. Inhibition of p38MAPK and CD137 signaling reduce dengue virus-induced TNF-a secretion and apoptosis. Virol $J$ 2013;10:105

25. Ascierto PA, Simeone E, Sznol M, Fu YX, Melero I. Clinical experiences with anti-CD137 and anti-PD1 therapeutic antibodies. 
Semin Oncol 2010;37:508-16.

26. Kim JO, Kim HW, Baek KM, Kang CY. NF-kappaB and AP-1 regulate activation-dependent CD137 (4-1BB) expression in T cells. FEBS Lett 2003;541:163-70.

27. Meyskens FL Jr, Buckmeier JA, Mc Nulty SE, Tohidian NB. Activation of nuclear factor-kappa B in human metastatic melanomacells and the effect of oxidative stress. Clin Cancer Res 1999;5: 1197-202.
Cite this article as: Harter PN, Blank AE, Weide B, Beschorner R, Bernatz S, Baumgarten P, Braczynski AK, Hattingen E, Ronellenfitsch MW, Schwarz $\mathrm{H}$, Mittelbronn $\mathrm{M}$. Tumor necrosis factor receptor superfamily member 9 is upregulated in the endothelium and tumor cells in melanoma brain metastasis. Neuroimmunol Neuroinflammation 2014;1(3):135-40.

Source of Support: Nil. Conflict of Interest: No.

Received: 14-07-2014; Accepted: 20-08-2014 